\title{
Curvature-specific color aftereffects
}

\author{
BORIS CRASSINI and RAY OVER \\ University of Queensland, St. Lucia, Queensland, Australia 4067
}

\begin{abstract}
Adaptation to convex and concave arcs in different colored light results in curvature-specific color aftereffects when arcs are later viewed in white light. In three experiments, it was shown that these color aftereffects often are partial (restricted to limited regions of the test arcs) rather than uniform, and in addition that aftereffects induced by exposure to arcs transfer to straight-line displays of particular orientation, and vice versa. These data were interpreted as evidence that arcs are processed in the visual system in relation to the orientations of local straight line approximations instead of on a global basis. In these terms, curvature-specific color aftereffects are merely complex forms of the orientation-specific color aftereffects first described by McCollough (1965).
\end{abstract}

There have been many recent demonstrations that adaptation to colored patterns results in color aftereffects that are spatially contingent, and in spatial aftereffects that are selective to wavelength. Thus, a vertical grating shown in white light appears blue and a horizontal grating looks orange following a period of exposure to vertical lines in orange light and horizontal lines in blue light (McCollough, 1965). Color aftereffects that are contingent on the direction of image motion (Hepler, 1968), spatial frequency (Lovegrove \& Over. 1972), and visual curvature (Riggs, 1973) have also been described. Wavelengthselective spatial aftereffects have been reported in the perception of contour orientation (Held \& Shattuck, 1971), image motion (Favreau, Emerson, \&Corballis, 1972), and spatial frequency (Stromeyer, 1972). In the case of orientation, for example, a vertical grating appears tilted clockwise following exposure to counterclockwise-tilted lines, and the distortion is greater when the inspection and test lines are shown in the same colored light than when they are shown in different colored light.

Both classes of contingent aftereffect have been attributed to selective adaptation of visual detectors with chromatic as well as spatial tuning. In the case of orientation-specific color aftereffects, McCollough (1965) supposed that there were separate detectors for blue-vertical and orange-vertical lines, and that vertical lines shown in white light were normally signaled by a balance between the two mechanisms. She further supposed that the class of detector excited during the inspection period was in a suppressed state for a period of time afterwards; the vertical lines in white light thus appeared colored because one of the mechanisms by which they were normally signaled was adapted while the other was not.

In terms of the above explanation, the different

This study was supported in part by an award from the Australian Research Grants Committee. Requests for reprints should be sent to Boris Crassini. Department of Psychology. University of Queensland, St. Lucia, Australia, 4067. colored patterns shown during the inspection period must be represented in the visual system by detectors with not completely identical sensitivity for induction of spatially linked color aftereffects (subsequently referred to as the McCollough effect). Exposure to the different color-spatial combinations would adapt both sets of detectors by which the test stimulus was signaled if this condition was not met; thus, the adaptation would not be selective, and the imbalance in neural response required for occurrence of the aftereffect would not pertain. The extent of spatial difference between the inspection stimuli is, in fact, critical for induction of the McCollough effect. and the spatial selectivity that occurs is consistent with tuning functions for feature detectors obtained using other psychophysical paradigms (particularly masking) and by direct electrophysiological measurement. Data from the former source suggest that orientation detectors are tuned over an angular range of \pm 10 -20 deg. and spatial frequency detectors over \pm 1 octave (see Blakemore \& Campbell, 1969). The spatial selectivity of the McCollough effect is consistent with these values. Fidell (1970) found that exposure in different colored light to lines that differ in orientation by only $11 \mathrm{deg}$ does not induce the McCollough effect. while a separation of $22 \mathrm{deg}$ produces desaturated aftereffects, and a difference of 45 deg results in saturated aftereffects. Similarly. Lovegrove and Over (1972) found that vertical graltings show $n$ in different colored light must differ in spatial frequency by at least 1 octave for periodicityspecitic color aftereffects to be obtained.

The ease with which the McCollough effect is induced can thus be predicted, within a selective adaptation model. from data on the spatial selectivity of feature detectors. Conversely, it should be possible to use the McCollough paradigm to establish the spatial selectivity of feature detectors when tuning functions are not available from other sources. The experiments reported in the present paper examine curvature-specific color aftereffects within both contexts. 
Riggs (1973) has reported that curvature-specitic color aftereffects result from exposure to convex arcs in red light and concave ares in green light. Following the inspection period. subjects report that convex lines shown in white light appear green and concave lines look red. The most saturated aftereffects always occurred at the extreme test curvatures irrespective of the degree of curvature of the inspection ares. Riggs attributed these aftereffects to selective adaptation of vistal detectors responsive to wavelength and curvature. He suggested that such detectors have strong differential tuning for direction of curvature (convex us. concave), and within a given direction detectors do not differ in terms of the degree of curvature at which each is most active but instead "simply respond more vigorously as radius of curvature is diminished to some optinum value" (Riggs. 1973. p. 1071).

Curvature masking functions (Crassini \& Over, 1975) contradict this proposition. There is no clear discontinuity in masking as the curvature of test lines changes from convexity to concavity. Although masking is broadly selective to the degree of curvature, for both slight and extreme curves the threshold for detection of an are is maxinally elevated by exposure to lines of identical radius. It would be expected from such data that color aftereffects would be maximally saturated when the inspection and test ares are identical in radius, show extensive gencralization along the curvature dimension, and evell transfer from convex to concave. As the masking data suggest that curvature detecting mechanisms are broadly tuned. it should in addition be impossible to induce color aftereffects when the ares shown in diflerent colored light differ only slightly in curvature fand thereby have considerable overlap in neural representation). However, inspection of ares that differ greatly in curvature (represented largely by independent mechanisms) should produce strong colored aftereffects.

Experiment I was designed to test these predictions. thereby providing information about spatial selectivity in the curvature McCollough effect. The measure of interest was the frequency of color reports to a series of test arcs differing in radius. It was predicted that frequency distributions would depend on whether the colored arcs viewed during adaptation were similar or markedly different in curvature. However, it was noted in preliminary testing that color aftereffects often did not have a uniform appearance across the test display. In many cases, only part of the test pattern (typically the top and the bottom) appeared colored or the center region differed in color appearance from the top and bottom. Fragmentation and variegation were found to vary systematically with the curvature relationship between the adaptation and test ares, and for this reason it was decided that the relevant dependent variable for examination of spatial selectivity of the aftereffect was the frequency of color reports in local segments of the test display rather than across the total figure.

Riggs (1973), in his report of the curvature $\mathrm{McC}$ cllough effect. made no reference to partial or variegated color aftereffects. The data reported in Experiments I and II replicate some of Riggs' findings if spatial selectivity in the frequency of color reports is considered without regard to fragmentation. How ever, quite different conclusions about the spatial selectivity of curvature-detecting mechanisms in human vision result when attention is given to the spatial localization of the aftereffect. In fact, the data make us think that the visual system analyzes the curvature of an arc by a series of local straight-line approximations instead of on a global basis.

\section{EXPERIMENT I}

\section{Method}

Subjects. Twelve undergraduate students with normal or corrected-to-normal vision were tested. All subjects were naive with respect to the McCollough effect, and they were screened for anomalies in color vision by the Ishihara test.

Apparatus and Procedure. Aftereffects were measured by requiring subjects to report whether color was visible at different locations within a test pattern viewed in white light following alternate exposure to arcs of one curvature in red light (Wratten Filter 26) and arcs of another curvature in green light (Wratten Filter 55). The adaptation and test stimuli (see Figure 1) were square-wave gratings of arcs with the following properties: chord orientation, vertical; spatial frequency, 2.5 cycles/deg; dimensions, $7 \mathrm{deg} 48 \mathrm{~min} \times 3 \mathrm{deg} 54 \mathrm{~min}$ visual angle; contrast, .7; space-average luminance, $23 \mathrm{~cd} / \mathrm{m}^{2}$. Three pairs of adaptation curvatures ${ }^{1}$ were employed: both slightly curved $(10-$ and 20 -deg radius), both extremely curved (2- and 2.5 -deg radius), and one extremely curved and the other slightly curved (2- and 20-deg radius). With in a given session. the pair of arcs were always both convex or both concave; this variable was counterbalanced across subjects, as was the color in which each of the arcs was displayed. Each test stimulus comprised two laterally arranged gratings of arcs (each subtending $3 \mathrm{deg} 54 \mathrm{~min} \times 3 \mathrm{deg} 54 \mathrm{~min}$ in visual angle). Thirty-two combinations were constituted by pairing each of the adaptation curvatures with a grating of straight lines and with gratings of convex and concave arcs of $2-., 2.5-, 3-, 4-, 6-, 10 \cdot$, and 20 -deg radius, with lateral position counterbalanced for all combinations.

The adaptation gratings were shown in alternation for $5 \mathrm{sec}$ at a time, with a dark interval of $1.5 \mathrm{sec}$ between presentations. The subjects were instructed to inspect the center of the display. The total adaptation period was $30 \mathrm{~min}$. and this was interrupted every 5 min to allow test measures to be taken. Following $30 \mathrm{sec}$ in darkness to allow for dissipation of afterimages, 16 of the test combinations were shown, each for $15 \mathrm{sec}$. The subject was required to mark on score sheets the presence and location of any color that appeared on a test grating.

Each subject was tested over three sessions (separated by several days because of evidence that the McCollough effect dissipates slowly) to enable measures to be obtained using the three pairs of adaptation curvatures.

\section{Results and Discussion}

The results shown in Figure 2 indicate the percentage frequency of uniform and partial color reports given on test trials as a function of the relative 


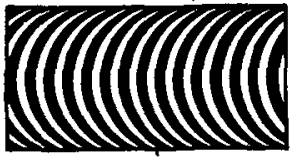

A

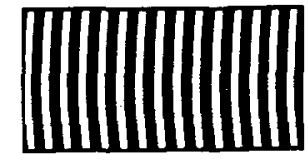

B
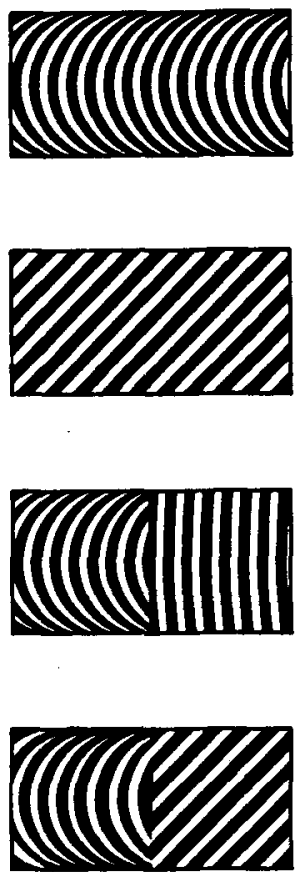

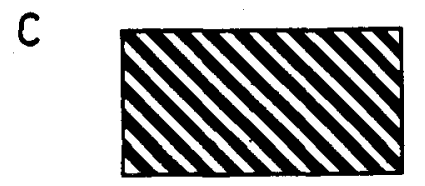

D

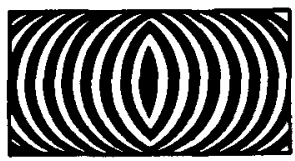

E

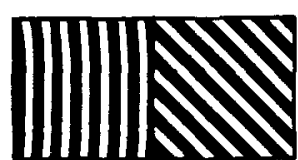

Figure 1. Examples of adaptation (1A, 1B, 1C) and test (1D, 1E) stimuli used in Experiments I, II, and III. Figure $1 \mathrm{~A}$ shows a pair of gratings of concave arcs, both strongly and slightly curved (Experiment I); $1 \mathrm{~B}$ shows convex and concave gratings of strongly curved ares (Experiments II and III); 1C shows right and left oblique straight-line gratings (Experiment III). The test stimuli (1D, 1E) are made up of lateral pairs of both curvilinear and rectilinear gratings. For a full description of stimulus parameters. see text.

direction and degree of curvature of the adaptation and test arcs. The variables that were counterbalanced in the design of the experiment (adaptation to convex or concave arcs, adaptation to the less curved arcs in red or green light) did not exert differential effect on the type or distribution of color reports. and data were therefore pooled without regard for these variables. A color report was classitied as uniform if the appropriate color was reported as appearing homogeneously distributed over the complete grating. and as partial if the color was localized to specitic regions of the grating. In the latter case, the appropriate color was invariably reported in horizontal bands across the top and bottom regions of the grating. with the center colorless or sometimes tinged with the complementary hue.

Three features of the results should be noted. First. very few curvature-specific aftereffects were induced by alternate exposure to arcs that were both extremely curved (Figure 2B) or both slightly curved (Figure 2C). However, after inspection of slightly curved and extremely curved gratings in differently colored light, most test arcs were reported as colored (Figure $2 \mathrm{~A}$ ). The induction curvature pairings under consideration were selected on the basis of masking data (Crassini d Over. 1975), and in this context the results suggest that it was only under the conditions represented in Figure $2 \mathrm{~A}$ that the induction pair differed sufficiently in spatial value for adaptation to be selective. Second. the frequency distribution of color reports was not symmetrical about the curvature values used during induction. Almost $80 \%$ of the extremely curved test arcs were seen as colored, whereas only $30 \%$ of slightly curved arcs were so reported. This inequality is not consistent with the proposition of complete independence of neural representation of the curvature values used to induce the color aftereffects. Further support for this conclusion is provided by the third feature of the results, viz. the difference in the type of aftereffect reported with extremely curved and slightly curved test ares. Gratings of weak curvature were exclusively seen as uniformly colored. However, as the radius of the test arcs decreased. the frequency of uniform responses also decreased and the number of partial color reports increased until. with extremely curved test arcs, partial responses were almost always given.

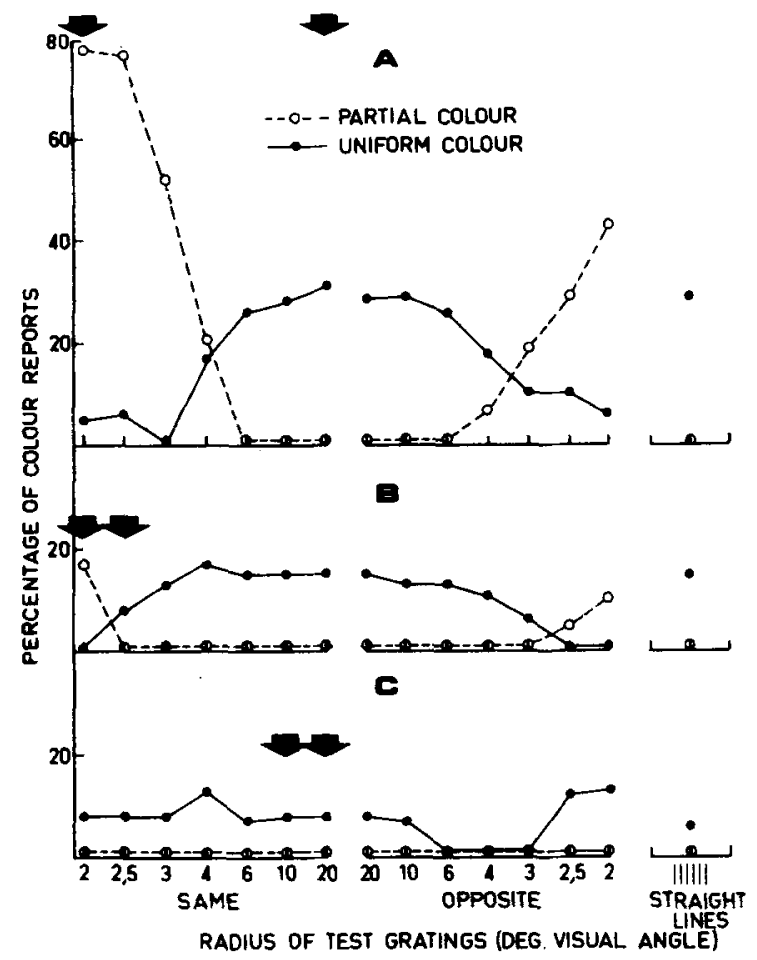

Figure 2. Percentage of color reports at different test curvatures following adaptation to 2 - and 20-deg arcs (A), 2- and 2.5-deg ares (B), and 10- and 20-deg ares (C) of the same direction of curvature. 
A complicating factor arises with test arcs of opposite direction of curvature to the inducing gratings. With these arcs, the trends in relation to the type of aftereffect reported by subjects were similar to those with test arcs of the same direction of curvature as the induction pair, but the frequency of appropriate aftereffects was reduced and the number of inappropriate responses increased.

The unequal distribution of color responses and the occurrence of partial aftereffects cannot be explained in terms of selective adaptation of curvature-sensitive detectors tuned to wavelength; such an account implies that induced color will be uniform across the test display and should occur equally for test stimuli having either of the spatial values used during induction. Partial aftereffects might be expected, however, if arcs are processed in the visual system by reference to the orientations of their straight-line approximations rather than on a global basis. Tangents along the perimeter of a slightly curved arc with vertical chord vary only slightly around vertical, but tangents at the top, center, and bottom of the arc differ markedly in orientation as curvature increases. The property that slightly and extremely curved arcs have in common is that tangents at the center have the same orientation in the two cases. In these terms, a curvature-specific color aftereffect represents the orientation-specific color aftereffects induced at different locations across the test display. Therefore, color aftereffects would be uniform if all orientations by which the test arc is signaled have been adapted with respect to only one color, while partial aftereffects would occur if some orientations have been adapted in relation to one color and other orientations to both colors or neither color. The basis for supposing that curvature is analyzed by orientation detectors is further considered in the General Discussion.

\section{EXPERIMENT II}

In Experiment I, the pairs of arcs presented in different colored light during inspection differed in radius but had the same direction of curvature, whereas in the study by Riggs (1973) the adaptation arcs were identical in radius but different in direction of curvature. Experiment II considers whether partial color aftereffects are also induced under the latter condition. The presence and location of color in the test displays was measured following exposure to convex and concave arcs of the same radius $(2,4$, or $20 \mathrm{deg}$ ) in different colored light.

\section{Method}

Subjects. Eight undergraduate and two postgraduate students without knowledge or experience of the McCollough effect were tested. Each subject had normal vision, and was screened for defects in color vision by the Ishihara test.
Apparatus and Procedure. The dimensions and general properties of the stimuli were as has been described for Experiment I. Each subject was tested under three adaptation conditions (different radius values) over sessions that were separated by several days. In a single session, the subject adapted to convex and concave ares of the same radius $(2,4$, or $20 \mathrm{deg})$ in different colored light. Convex arcs were shown in red light and concave ares in green light for half the subjects, and the opposite combination was used for the remainder. The test stimuli were laterally arranged pairs of gratings representing the combination of the two adaptation curvatures with convex and concave arcs of radius $2,2.5,3,4,6,10$, and $20 \mathrm{deg}$, as well as with a grating of straight lines. The aftereffect was measured following each 5-min adaptation, and 16 test combinations were shown for $15 \mathrm{sec}$ each during every test period. The subject's task was to mark on score sheets the presence and location of any color visible on the test gratings. The procedure was similar in all other respects to that described in Experiment $\mathrm{I}$.

\section{Results and Discussion}

The percentage frequency of uniform and partial color reports on test trials is shown in Figure 3 as a function of the direction and degree of curvature of the adaptation and test arcs. The results show clear spatial selectivity in induction of both uniform and partial color reports. Adaptation to slightly curved ares produced few aftereffects (Figure $3 \mathrm{C}$ ), whereas alternate exposure to convex and concave arcs of extreme curvature resulted in a large number of color reports (Figure $3 \mathrm{~A}$ ). The type of aftereffect varied

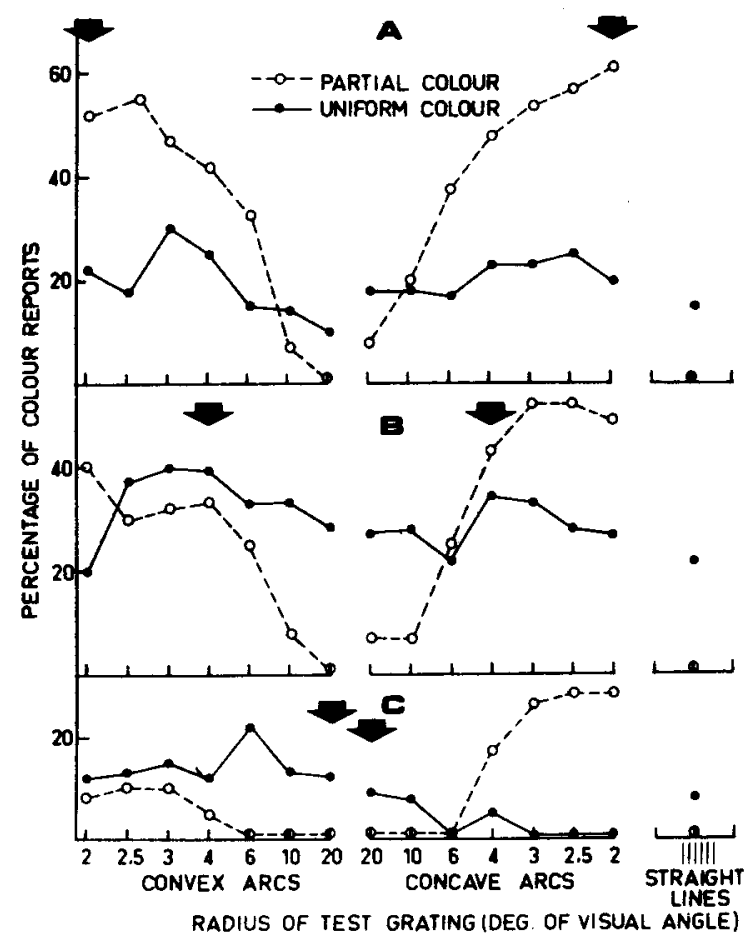

Figure 3. Percentage of color reports at different test curvatures following adaptation to 2-deg convex and 2-deg concave arcs (A). 4-deg convex and 4-deg concave ares (B), and 20-deg convex and 20-deg concave ares $(C)$. 
with the curvature of the test grating in the manner found in Experiment I; partial reports were given to extremely curved test ares but never to slightly curved test arcs, and uniform color was rarely seen. In the case of partial aftereffects, the top and bottom of the test arcs always appeared complementary to the color of the adaptation arcs with the same direction of curvature and the center appeared colorless or sometimes tinged with the adaptation hue. Inspection of noderately curved arcs (Figure $3 \mathrm{~B}$ ) produced the same trends, except that there was a higher frequency of uniform color reports and a lower frequency of partial color reports (restricted to moderate and extremely curved test arcs).

The present data are in part consistent with results reported by Riggs (1973) if the frequency of color reports is considered without regard to type. In these terms, aftereffects were more readily induced as the difference in curvature between the inspection arcs increased, and it could be argued that this result has at its basis improved selectivity in adaptation of color-tuned curvature detectors under the conditions of large stimulus difference. However, only a small number of aftereffects were induced by inspection of weakly curved gratings, contrary to data reported by Riggs (1973). Moreover, attereffects were not located uniformly across the test display, and in explaining curvature-specific color aftereffects it is necessary to outline why induced color is limited to parts of the test display in a manner dependent on the relative degree and direction of curvature of adaptation and test arcs. Partial aftereffects are later considered by reference to adaptation of detectors that process the orientations of straight-line segments by which an arc can be approximated.

\section{EXPERIMENT III}

In the classical McCollough effect (McCollough, 1965), exposure to straight lines in colored light produces orientation-specilic color aftereffects when straight lines are subsequently viewed in white light. The proposition that ares are represented in the visual system in relation to the orientations of straight-line approximations to the arcs suggests that color aftereffects would occur when arcs are viewed in white light following adaptation to colored straight-line gratings. However, only those parts of the test arcs with tangents of approximately the same orientation as the straight-line induction gratings should appear colored. Moreover, because the orientational information provided by the upper and lower regions of extremely curved ares is almost orthogonal, the apparent color reported in these regions should not be the same (as in previous partial color reports), but should be opposite. In other words, after inspection of straight-line induction gratings, curved test arcs should be reported as partially colored, with the upper and lower regions of the ares in complementary hues. In addition, aftereffects induced by exposure to ares in colored light should transfer to straight-line test gratings show $n$ in white light, with the color restricted to the locations within the visual field that received similar orientation information under the adaptation and test conditions. The straight-line test gratings should be reported as partially colored, with the upper and lower regions seen in complementary hues associated with the opposite McCollough effects being simultaneously induced by the upper and lower regions of the curved induction gratings. These expectations are tested in Experiment III.

\section{Method}

Subjects. Ten undergraduate students with normal color and spatial vision were tested. No subject had had previous experience with the McCollough effect.

Apparatus and Procedure. Each subject was tested in two sessions separated by several days. In one session, convex arcs of 2-deg radius in red (or green) light and concave ares of 2-deg radius in green (or red) light were shown alternately every $5 \mathrm{sec}$ over $30 \mathrm{~min}$. The adaptation period was interrupted every $5 \mathrm{~min}$ to allow presentation of test stimuli in white light. The 36 test stimuli in the experiment comprised lateral pairings of gratings of left-oblique and right-oblique straight lines with each other, with vertical straight lines, and with convex and concave ares of 2-, 2.5-, 3-, 4-, 6-. 10-, and 20-deg radius. During a test period, 18 of these stimuli were shown successively for $15 \mathrm{sec}$ each, and the subject was required to indicate on a score sheet the presence and location of any color visible on the gratings. In the other session, the color appearance of the same test stimuli was judged after adaptation to left-oblique and right-oblique straight lines shown in different colored light (red, green). The dimensions, spatial frequency, contrast, and space-average luminance of the gratings were as described for Experiment $\mathbf{I}$.

\section{Results and Discussion}

The percentage frequency of test trials on which color reports were given is shown separately for adaptation to arcs (Figure 4A) and straight lines (Figure 4B). The distribution of color reports when curved lines were seen after exposure to curved lines matches the results obtained in Experiment II, and the standard McCollough effect was obtained when straight lines were viewed following adaptation to colored straight lines. The data of interest relate to transfer of color aftereffects between curved and straight lines. Figure 4A show's that adaptation to colored arcs resulted in color reports when left-oblique and right-oblique straight lines were subsequently shown, but that vertical straight lines did not appear colored. The aftereffects were predominantly of the "opposite color" partial category; exposure to red convex and green concave arcs resulted in a red aftereffect in the upper region and a green aftereffect in the lower region of a right-oblique grating, and the reverse was found with a left-oblique grating. Similar partial reports were also given when arcs were shown as test stimuli 


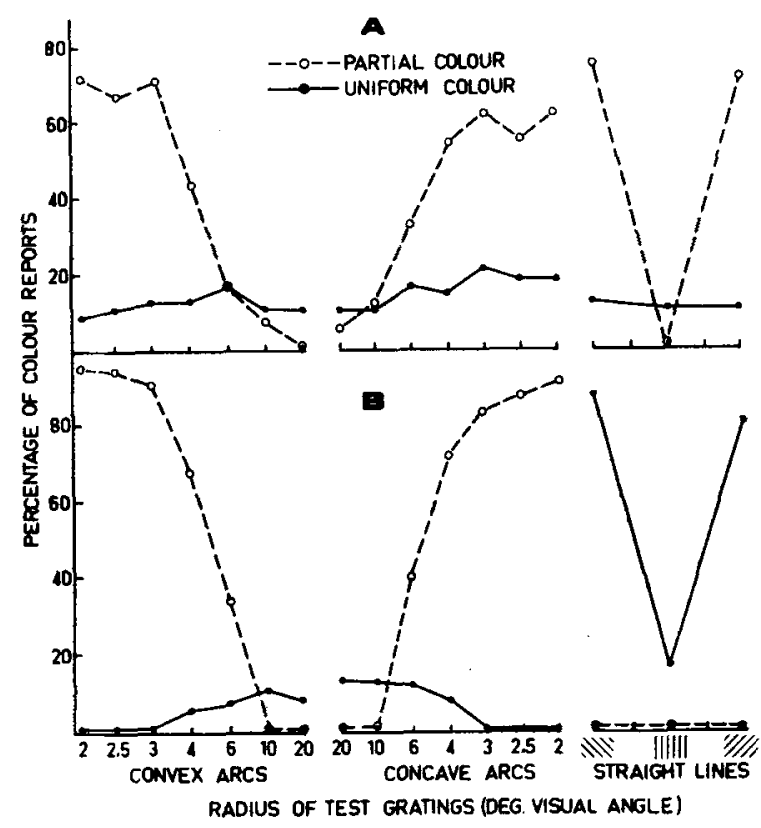

Figure 4. Percentage of color reports at different test curvatures following adaptation to curved-line (A) and straight-line (B) gratings.

following adaptation to right-oblique and left-oblique gratings, and the frequency with which the aftereffects were induced varied with the curvature of the arcs (see Figure 4B). The predictions concerning transferability of color aftereffects between linear and curvilinear gratings are clearly confirmed by these data. Further support is thus provided for the proposition that straight and curved contours are processed by common mechanisms in the visual system.

\section{GENERAL DISCUSSION}

The present experiments have demonstrated that exposure to colored ares results in curvature-specific color aftereffects when test lines are subsequently show $n$ in white light. However, the properties of the aftereffects differed from those earlier described by Riggs (1973). In particular, the test arcs often had partial rather than uniform color appearance, and there was spatial selectivity in the frequency with which each class of report was given. Uniform color reports were found with slightly curved test lines and partial color reports with extremely curved test lines following exposure to colored arcs differing in radius by a critical amount (Experiment I) or in direction of curvature (Experiment II).

It was suggested earlier that curvature-specific color aftereffects are in essence a compound form of the orientation-specific color aftereffects first described by McCollough (1965). The proposition is that the visual system analyzes curvature in relation to the orientation of local straight-line approximations along an arc rather than on a global basis. In these terms, a curvature-specific color aftereffect reflects the orientation-specific color aftereffects induced at different sites within the test display. Thus color aftereffects would be uniform if all local orientations by which the test arc is signaled have been adapted with respect to only one color, while partial aftereffects would occur if some local orientations have been adapted in relation to one color and other local orientations to both colors or to neither color. This interpretation is supported by the results of Experiment III; adaptation to colored arcs resulted in aftereffects when straight-line patterns were later shown in white light (and vice versa) but the color appearance was restricted to the part of the test display that provided the same local orientation information as the adaptation stimulus.

There are other data that are consistent with the claim that curvature is not a primary dimension of visual analysis. MacKay and MacKay (1974) have shown that curvature-specific color aftereffects are not induced when the subject has made pursuit movements along the chord of the adaptation arc during the inspection period. This condition should yield aftereffects if curvature is processed on a global basis, but not if visual analysis is undertaken with reference to local orientation information. In the latter terms, the inspection condition would produce nonselective adaptation of color-tilt detectors in that any given part of the retina would be exposed to a wide range of orientation values under each color condition during the inspection period. Blakemore and Over (1974) have similarly shown that pursuit eye movements along the chord of the adaptation arc eliminate the spatial aftereffects of curvature.

Data on curvature-specific contour masking, aftereffect, and illusion induced with fixation (Crassini \& Over, 1975) are open to interpretation in terms of selective adaptation or inhibition of global curvature detectors. The present results suggest, however, that such data can be more profitably considered in relation to operations by which the visual system processes local orientation information.

\section{REFERENCES}

Blakemore, C. \& Campbell, F. W. On the existence of neurones in the human visual system selectively sensitive to the orientation and size of retinal images. Journal of Physiology, 1969. 203, $237-260$.

Blakemore, C., \& Over. R. Curvature detectors in human vision? Perception, 1974, 3, 3-7.

Crassini, B., \& Over, $\dot{R}$. Masking, aftereffect, and illusion in visual perception of curvature. Perception \& Psychophysics, 1975, 17, 411-416.

Favreau, O., Emerson, V., \& Corballis, M. C. Movement aftereffects contingent on color. Science, 1972, 176, 78-79.

Fidel, L. S. Orientation specificity in chromatic adaptation 
of human "edge" detectors. Perception \& Psychophysics, 1970. 8. 235-237.

Held. R.. \& Shattuck, S. R. Color-and edge-sensitive channels in the human visual system: Tuning for orientation. Science, 1971, 174, 314-316.

HePler, N. Color: A motion-contingent aftereffect. Science, $1968,162,376-377$.

Lovegrove. W. J.. \& Over, R. Color adaptation of spatial frequency detectors in the human visual system. Science. 1972, 176. $541-543$.

McCollough. C. Color adaptation of edge detectors in the human visual system. Science. 1965, 149, 1115-1116.

MACKAY, D. M., \& MACKAY, V. Do curvature-contingent chromatic aftereffects require "detectors for curvature"? Vision Research, 1974, 14, 1285-1287.

Riggs, L. A. Curvature as a feature of pattern vision. Science, 1973, 181, 1070-1072.
Stromeyer, C. F. Edge-contingent color after-effects: Spatial frequency specificity. Vision Research. 1972, 12. 717-733.

\section{NOTE}

1. The stimuli used in the experiments were selected to cover a range of curvatures from a convex arc ( $)$ ) almost forming a semicircle, through vertical straight, to a concave arc (c) of the same dimensions. The stimulus series covered approximately equal steps of curvature defined as the reciprocal of radius. However, following the convention adopted by Riggs (1973) and others, curvature values are expressed throughout this paper in terms of radius (degrees of visual angle) rather than the reciprocal of radius.

(Received for publication August 8, 1974; revision received December 5,1974 .) 